\title{
AUTOMATIC AND SAMPLING-FREE PARAMETRIC MODEL ORDER REDUCTION OF VIBRO-ACOUSTIC SYSTEMS
}

\author{
S. van Ophem ${ }^{1,2, a}$, E. Deckers ${ }^{1,2}$ and W. Desmet ${ }^{1,2}$ \\ ${ }^{1}$ KU Leuven, Department of Mechanical Engineering, Celestijnenlaan 300 B, B-3001, Heverlee, \\ Belgium \\ ${ }^{2}$ Flanders Make, DMMS core lab, Belgium \\ ${ }^{a}$ Corresponding author: sjoerd.vanophem@kuleuven.be
}

Key words: Parametric model order reduction, Vibro-acoustics, Low-rank

\begin{abstract}
Recently, a novel parametric model order reduction formulation has been derived for vibroacoustic systems that allows for the reduction of systems with low-rank parametric changes [1]. This scheme does not require sampling of the parameter space, in contrast to conventional parametric model reduction techniques. This means that a single reduction basis, obtained with conventional non-parametric model order reduction schemes, can be used for a wide range of parameter values. This is done by rewriting the system in a non-parametric form, in which the low-rank contributions act as inputs. A disadvantage of this scheme is that the size of the input matrix scales with the amount of chosen parameters, leading to a potentially large reduced basis when many parameters are considered.

Therefore, in [2] an automatic Krylov reduction scheme has been proposed that utilizes the similarity in the reduced bases for inputs which are spaced closely together to still get a small reduced basis with a large number of inputs. This is done by using a combination of block second order Arnoldi with a singular value decomposition acting on the resulting basis. The algorithm includes an error estimator that uses a complementary approximation to calculate the error. The main advantages of this algorithm as compared to the commonly used iterative rational Krylov approach [3] are that only a small amount of system inversions are required and that the final reduced order model has the desired predefined relative error in the specified frequency band.
\end{abstract}

In this paper the automatic Krylov reduction scheme and low-rank parametric model order reduction approach are combined and a suitable error estimator is derived, to arrive at compact but accurate parametric reduced order models. The effectiveness is shown with several examples.

\section{INTRODUCTION}

In the last few decades the increase of computational power has lead to the rise of computer modeling techniques to predict the vibro-acoustic behavior of machines, consumer products, etc. For low to mid frequencies the usage of element based techniques, such as the Finite Element Method (FEM) and Boundary Element Method (BEM) has become the standard in both industry and academia. However, although computing power has increased immensely, the requirement of industry and academia on what these models should be able to compute has grown even more. This means that even today solving these 
models can take significant resources and computing time. Especially when many models have to be evaluated, such as is the case when parameter optimization is performed, it is not uncommon to have to wait for weeks for the optimizer to converge.

To overcome these problems, in recent decades a significant research effort has been performed on Model Order Reduction (MOR). The goal of MOR is to reduce the system size significantly, while keeping the resulting Reduced Order Model (ROM) accurate enough to calculate the solution with the desired accuracy. When also parametric variations have to be retained in the resulting ROM this is called parametric Model Order Reduction (pMOR) [4].

The application of MOR on vibro-acoustic systems comes with specific challenges, such as time-stability and the modeling of parametric variations that are related to the physical system, such as structural boundary conditions, a change of material properties, etc [5]. Recently, a novel reduction scheme for lowrank parametric variations in vibro-acoustic systems was derived [1], using earlier derived techniques for first order system as starting point [6], [7]. The technique from [1] allows for the modeling of lowrank parametric variations, such as those that appear for the application of boundary conditions or the placement of local stiffeners/masses [8]. Furthermore, this technique does not rely on a-priori parameter sampling, in contrast to other common pMOR schemes. Thus, the reduced basis can be pre-calculated once and will be yalid for all parametric variations. Recently, also an alternative low-rank formulation has been derived [10], that will be extended and investigated in more detail in this paper for second order systems in Sec. 2. Since the authors of [10] mention several potential advantages, as compared to other approaches, a comparison between this approach and the formulation in [1] is done in Sec. 3, specifically

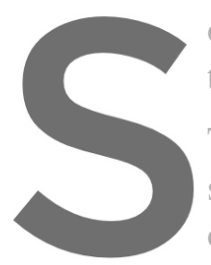
on stability and accurac to equivalent reduced or The proposed low-rank side of the set of dynamic of the Krylov subspace. T
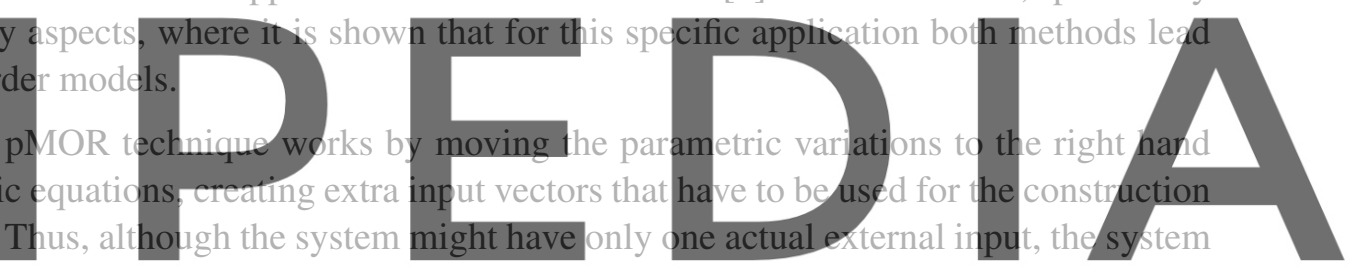

has to be treated as a multiple input system. In [2] an efficient automatic second order Arnoldi scheme is

[3] is that it automatically calculates the ROM to a predetermined accuracy in the desired frequency range with a minimum amount of expansion points, thus a relatively low computational cost as compared to IRKA. However, this algorithm relies on the calculation of an error estimator that checks the accuracy of the resulting reduced basis for each input individually. In the context of low-rank pMOR this demand on the model accuracy might be too strict and thus lead to too large ROMs. Therefore, in Sec. 4 an alternative error estimator is assessed that is specifically designed for second order systems with low-rank parametric changes. The resulting models are assessed with a representative vibro-acoustic example in Sec. 5.

\section{LOW RANK MODEL ORDER REDUCTION FORMULATION FOR VIBRO-ACOUSTIC SYSTEMS}

In the following section a possible way of formulating the vibro-acoustic system to allow for low-rank parametric changes is shown. This formulation is an extension to second-order systems of the formulation shown in [10]. An alternative formulation was previously shown in [1]. 


\subsection{Low rank pMOR formulation}

This formulation starts from the second order transfer function of a vibro-acoustic system in the Laplace domain (with Laplace variable s):

$$
H(s, \boldsymbol{p}, \boldsymbol{q}, \boldsymbol{r})=\mathbf{L}\left(s^{2} \mathbf{M}(\boldsymbol{r})+s \mathbf{C}(\boldsymbol{q})+\mathbf{K}(\boldsymbol{p})\right)^{-1} \mathbf{B}_{0} .
$$

The matrices $\mathbf{M}(\boldsymbol{r}), \mathbf{C}(\boldsymbol{q})$ and $\mathbf{K}(\boldsymbol{p}) \in \mathbb{R}^{n \times n}$ are parametrized mass, damping and stiffness matrices, dependent on the parameter vectors $\boldsymbol{p}, \boldsymbol{q}$ and $\boldsymbol{r}$. It is assumed that the system is excited by one excitation pattern, thus the input vector is given by $\mathbf{B}_{0} \in \mathbb{R}^{n \times 1}$. $\mathbf{L} \in \mathbb{R}^{n_{o} \times n}$ is the output matrix with $n_{o}$ output locations. The parametric dependency of the system matrices is assumed to be affine and a sum of low-rank changes, thus:

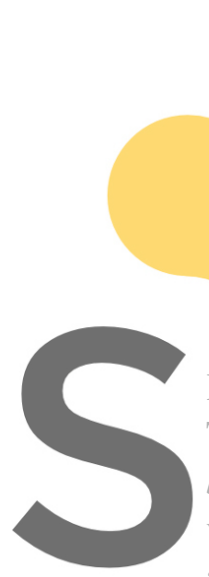

$$
\begin{aligned}
& \mathbf{K}(\boldsymbol{p})=\mathbf{K}_{0}+\sum_{k=1}^{v} p^{(k)} \boldsymbol{b}_{k} \boldsymbol{c}_{k}^{T}, \\
& \mathbf{C}(\boldsymbol{q})=\mathbf{C}_{0}+\sum_{l=1}^{\mu} q^{(l)} \boldsymbol{d}_{l} \boldsymbol{e}_{l}^{T},
\end{aligned}
$$$$
\mathbf{M}(\boldsymbol{r})=\mathbf{M}_{0}+\sum_{m=1}^{\xi} r^{(m)} \boldsymbol{f}_{m} \boldsymbol{g}_{m}^{T} .
$$

\section{$\mathbf{K}_{0}, \mathbf{C}_{0}$ and $\mathbf{M}_{0}$ are the part of the \\ The parametric dependen}

$b_{k}, c_{k}, d_{l}, e_{l}, f_{m}, g_{m}$

vector product leads to 1
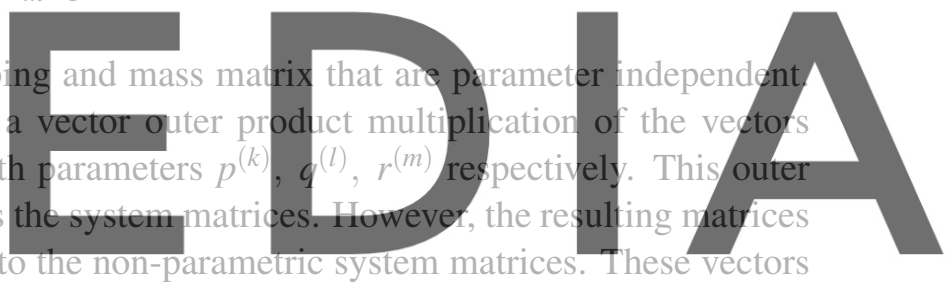

are assembled in the following matrices:

Register for free at https//www.scipedia.com to download the version without the watermark

$$
\begin{aligned}
\boldsymbol{b} & =\left[\begin{array}{lll}
b_{1} & \ldots & b_{v}
\end{array}\right] \in \mathbb{R}^{n \times v}, & \boldsymbol{c} & =\left[\begin{array}{lll}
c_{1} & \ldots & c_{v}
\end{array}\right] \in \mathbb{R}^{n \times v}, \\
\boldsymbol{d} & =\left[\begin{array}{lll}
d_{1} & \ldots & d_{\mu}
\end{array}\right] \in \mathbb{R}^{n \times \mu}, & \boldsymbol{e} & =\left[\begin{array}{lll}
e_{1} & \ldots & e_{\mu}
\end{array}\right] \in \mathbb{R}^{n \times \mu}, \\
\boldsymbol{f} & =\left[\begin{array}{lll}
f_{1} & \ldots & f_{\xi}
\end{array}\right] \in \mathbb{R}^{n \times \xi}, & \boldsymbol{g} & =\left[\begin{array}{lll}
g_{1} & \ldots & g_{\xi}
\end{array}\right] \in \mathbb{R}^{n \times \xi} .
\end{aligned}
$$

We continue by noting that the transfer function of a second order system, including possible parametric variations in the mass, stiffness and damping matrix, can be written as follows:

$$
H(s, \boldsymbol{p}, \boldsymbol{q}, \boldsymbol{r})=\mathbf{L}\left(s^{2} \mathbf{M}_{0}+s \mathbf{C}_{0}+\mathbf{K}_{0}-\mathbf{U D}(\boldsymbol{p}, \boldsymbol{q}, \boldsymbol{r}) \mathbf{W}^{T}\right)^{-1} \mathbf{B}_{0},
$$

in which $\mathbf{U}=\left[\begin{array}{lll}\boldsymbol{b} & \boldsymbol{d} & \boldsymbol{f}\end{array}\right], \mathbf{W}=\left[\begin{array}{lll}\boldsymbol{c} & \boldsymbol{e} & \boldsymbol{g}\end{array}\right]$, are matrices that indicate the positions of the low-rank changes. Define the matrix $\mathbf{D}(\boldsymbol{p}, \boldsymbol{q}, \boldsymbol{r})$ as follows:

$$
\mathbf{D}(\boldsymbol{p}, \boldsymbol{q}, \boldsymbol{r})=\operatorname{diag}\left(p_{1}+s q_{1}+s^{2} r_{1}, \ldots, p_{\tau}+s q_{\tau}+s^{2} r_{\tau}\right)
$$


with $\tau=\max (k, l, m)$. When either $k, l$ or $m$ is smaller than $\tau$, parameter values that extend beyond the length of the parameter vector are set to zero. Following the approach in [10], Eq. (6) can be rewritten using the matrix inversion lemma. This lemma is defined as follows:

$$
(\mathbf{E}+\mathbf{F G H})^{-1}=\mathbf{E}^{-1}-\mathbf{E}^{-1} \mathbf{F}\left(\mathbf{G}^{-1}+\mathbf{H E}^{-1} \mathbf{F}\right)^{-1} \mathbf{H} \mathbf{E}^{-1} .
$$

By choosing

$$
\begin{aligned}
& \mathbf{E}=s^{2} \mathbf{M}_{0}+s \mathbf{C}_{0}+\mathbf{K}_{0}, \\
& \mathbf{F}=-\mathbf{U}, \\
& \mathbf{G}=\mathbf{D}, \\
& \mathbf{I}=\mathbf{W}^{T},
\end{aligned}
$$

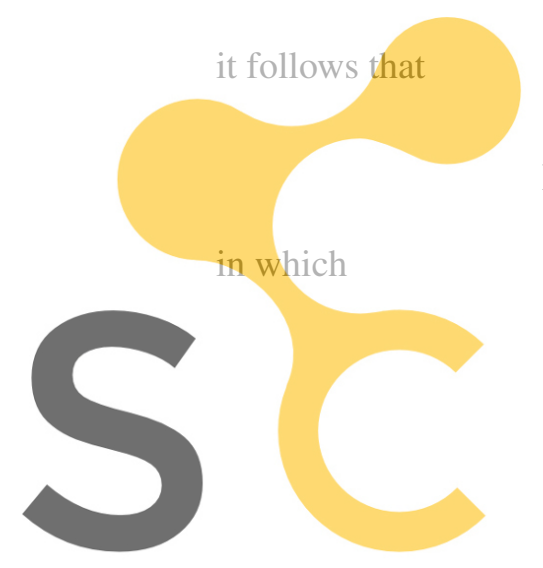

$$
\mathbf{H}(s, \boldsymbol{p}, \boldsymbol{q}, \boldsymbol{r})=\mathbf{H}_{1}(s)-\mathbf{H}_{2}(s)\left(\mathbf{D}^{-1}(\boldsymbol{p}, \boldsymbol{q}, \boldsymbol{r})+\mathbf{H}_{3}(s)\right)^{-1} \mathbf{H}_{4}(s),
$$
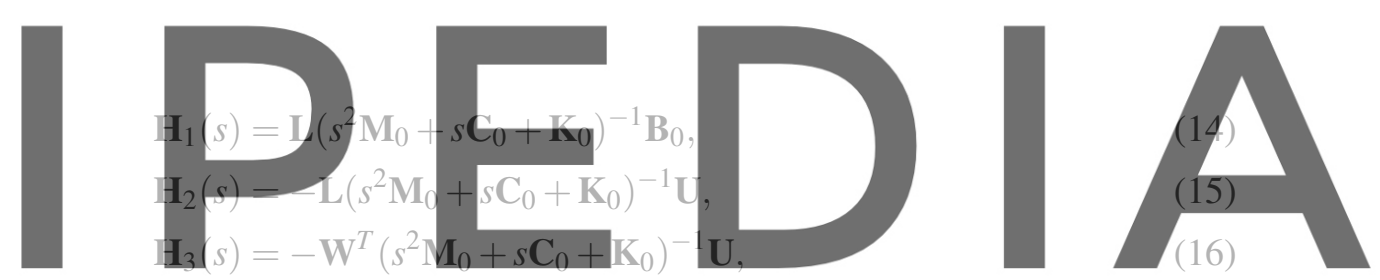

$\mathbb{H}_{4}(s)=\mathbf{W}^{T}\left(s^{2} \mathbf{M}_{0}+s \mathbf{C}_{0}+\mathbb{K}_{0}\right)^{-1} \mathbb{B}_{0}$.

(17)

\section{Register for free at https//www.scipedia.com to download the version without the watermark}

The individual transfer functions $\mathbb{H}_{1}(s)$ to $\mathbb{H}_{4}(s)$ are independent of parameters $\boldsymbol{p}, \boldsymbol{q}, \boldsymbol{r}$, thus can be redüced using non-parametric MOR techniques. Since for second order systems generally one-sided projections are used to calculate the reduction basis and it is assumed that $\mathbf{B}$ and $\mathbf{U}$ are linearly independent, this means that we can construct one reduction basis $\mathbf{V} \in \mathbb{C}^{n \times k}$ that can be used to calculate all of the reduced order transfer functions in Eq. (17), with

$$
\begin{aligned}
\hat{\mathbf{M}}_{0} & =\mathbf{V}^{H} \mathbf{M}_{0} \mathbf{V}, \hat{\mathbf{C}}_{0}=\mathbf{V}^{H} \mathbf{C}_{0} \mathbf{V}, \hat{\mathbf{K}}_{0}=\mathbf{V}^{H} \mathbf{K}_{0} \mathbf{V}, \\
\hat{\mathbf{B}} & =\mathbf{V}^{H} \mathbf{B}_{0}, \hat{\mathbf{U}}=\mathbf{V}^{H} \mathbf{U} \\
\hat{\mathbf{W}} & =\mathbf{W}^{T} \mathbf{V}, \hat{\mathbf{L}}=\mathbf{L V} .
\end{aligned}
$$

\section{Comparison between formulations}

As mentioned before, an alternative low-rank pMOR formulation is presented in [1]. This might lead to the question what the difference is between the two formulations. In [10] a comparison has been made 
between the two formulations for first order systems, but the points made in that paper do not necessarily hold for this specific case. In the following we will treat the stability and accuracy in more detail, since those points are mentioned in [10] as specific advantages of the alternative formulation.

\subsection{Stability}

When a time domain analysis is desired of a vibro-acoustic system, usually a one-sided projection is considered. This is done to preserve the definiteness properties of the full order system matrices, such that stability is preserved. For vibro-acoustic systems it has been shown how the use of a one-sided projection, in combination with a specific system formulation, leads to stability preservation [5]. Thus, both of the above approaches will lead to stable ROMs when the full order system is stable, a onesided projection is used, and the correct system formulation is used. Thus, under these assumptions stability is not an issue. It has to be remarked here that formulation in [1] lends itself better to a time domain analysis, since the formulation is derived in the time domain directly, while the new formulation is derived in the frequency domain.

\subsection{Accuracy}

In $[10]$ it is reported that another advantage of the new formulation is the ability to individually reduce ROMs $\mathbf{H}_{1}(s)$ to $\mathbf{H}_{4}(s)$, leading to possibly smaller ROMs. However, under the assumption that a onesided reduction is used, it is possible to split the ROMs of both formulations. To see why this is true, we will compare the Krylov subspaces that are spanned by the formulations in this paper and [1].
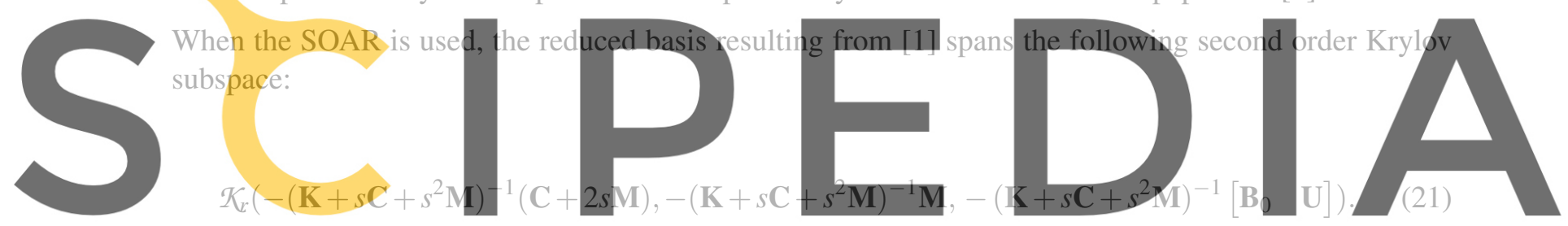

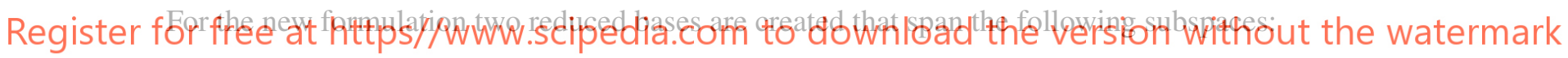

$$
\begin{gathered}
\mathscr{T}_{\mathbb{V}}\left(-\left(\mathbf{K}+s \mathbf{C}+s^{2} \mathbf{M}\right)^{-1}(\mathbf{C}+2 s \mathbf{M}),-\left(\mathbf{K}+s \mathbf{C}+s^{2} \mathbf{M}\right)^{-1} \mathbf{M},-\left(\mathbf{K}+s \mathbf{C}+s^{2} \mathbf{M}\right)^{-1} \mathbf{B}_{0}\right), \\
\mathcal{K}_{x}\left(-\left(\mathbf{K}+s \mathbf{C}+s^{2} \mathbf{M}\right)^{-1}(\mathbf{C}+2 s \mathbf{M}),-\left(\mathbf{K}+s \mathbf{C}+s^{2} \mathbf{M}\right)^{-1} \mathbf{M},-\left(\mathbf{K}+s \mathbf{C}+s^{2} \mathbf{M}\right)^{-1} \mathbf{U}\right) .
\end{gathered}
$$

Since a one-sided projection is used, transfer functions $\mathbf{H}_{1}$ and $\mathbf{H}_{3}$ are calculated with the same reduction basis, while $\mathbf{H}_{2}$ and $\mathbf{H}_{4}$ are calculated with another reduction basis. Since the input matrix $\left[\begin{array}{ll}\mathbf{B}_{0} & \mathbf{U}\end{array}\right]$ consists of linearly independent input vectors, the reduced bases $\mathbf{V}_{1}, \mathbf{V}_{3}$ for these input vectors can be calculated individually and then recombined in a later stage to calculate the final reduced basis:

$$
\mathbf{V}_{\text {tot }}=\operatorname{orth}\left(\left[\begin{array}{ll}
\mathbf{V}_{1} & \mathbf{V}_{2}
\end{array}\right]\right)=\operatorname{orth}\left(\left[\begin{array}{ll}
\mathbf{V}_{3} & \mathbf{V}_{4}
\end{array}\right]\right) .
$$

This basis spans the following subspace:

$$
\mathcal{K}_{r}\left(-\left(\mathbf{K}+s \mathbf{C}+s^{2} \mathbf{M}\right)^{-1}(\mathbf{C}+2 s \mathbf{M}),-\left(\mathbf{K}+s \mathbf{C}+s^{2} \mathbf{M}\right)^{-1} \mathbf{M},-\left(\mathbf{K}+s \mathbf{C}+s^{2} \mathbf{M}\right)^{-1}\left[\begin{array}{ll}
\mathbf{B}_{0} & \mathbf{U}
\end{array}\right]\right)
$$


Thus, both methods span the same subspace and can be calculated with the same SOAR procedure. However, to remove possible redundancy in the reduced basis an singular value decomposition (SVD) is performed on the combined reduction basis. Thus, it might be the case that the formulation in [1] even leads to smaller ROMs for a comparable accuracy, although differences will be minor.

\section{AUTOMATIC REDUCTION WITH ADJUSTED ERROR ESTIMATOR}

Whatever formulation is chosen for the calculation of the pMOR model, the resulting ROM has to be calculated with a possibly large set of input vectors. It is well known that the resulting ROM size scales with the amount of inputs. However, when the inputs are in close proximity and thus give a similar system response, the size increase might still be acceptable, because of redundant columns in the reduced basis. An automatic reduction algorithm that takes this redundancy into account has been derived in [2]. The algorithm uses an automatic error estimator to determine if for the chosen expansion point the amount of matched moments should be increased, which is based on a complementary approach. This means that two reduced bases are constructed, one with a slightly higher amount of matched moments than the other to locally simulate response of the full order model (FOM). Additionally, it uses an a-posteriori SVD to imitate the addition of extra input information when the error is estimated, again with different SVD thresholds for the two reduced bases. In [2] it was shown that this approach gives good error estimates. Nevertheless, this algorithm is made to perform optimally for systems in which all the extra inputs are actual external inputs and not only used to model low-rank parametric variations, as is the case

for this paper. Thus, the previous algorithm requires that for all the input vectors the relative error on the

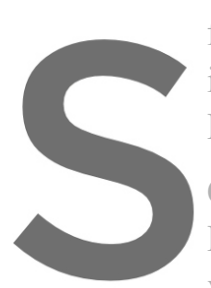
frequency response function is below a certain threshold. This might be unnecessarily influence of certain param ROMs that are larger than Concretely, two adjustrnents are Response Function (FRF) values are chosen randomly
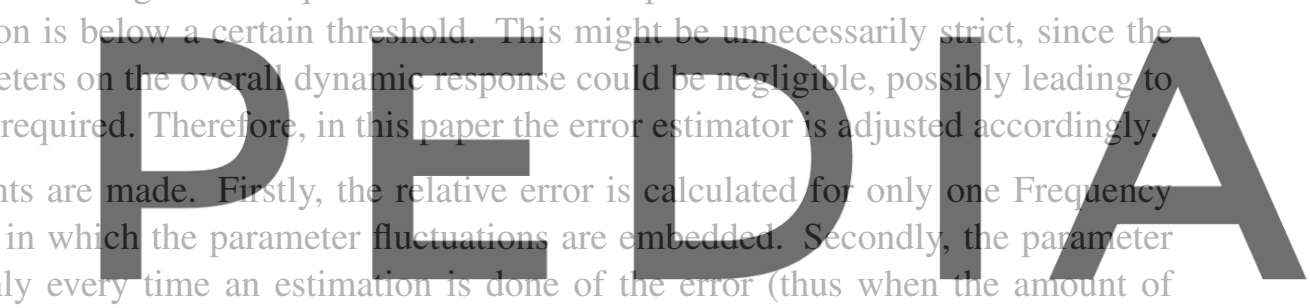

matched moments of a chosen expansion point is increased) to make sure that the relative error is below

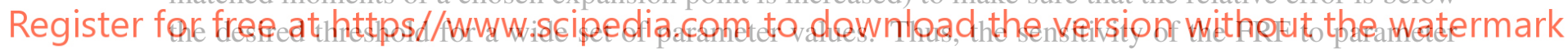

fluctuations is assessed. While this approach does not guarantee that the relative error will be below the desired threshold for full range of parameter values, it was found to be a reasonable approximation. The effectiveness of this approach is investigated in the following section.

\section{NUMERICAL VERIFICATION}

The optimized error estimator is validated with a vibro-acoustic numerical model that has previously been used in [8], see Figure 1 for a mesh of the FE model. The model represents the SoundBox, a vibroacoustic test rig at the KU Leuven Noise \& Vibration lab that consists of a concrete box with a clamped 3 $\mathrm{mm}$ thick aluminum plate on the front side of the box, see Figure 2. As written in [8], the exact boundary conditions of the plate, while approaching clamped conditions, are not describing the dynamics $100 \%$ accurately. Thus the plate is modeled with shell elements of which the exact boundary conditions are parameterized. In particular, the rotational stiffness of the left hand edge is parameterized. This leads to 30 independent parameters, thus requiring moment matching with 31 different input vectors using the ROM strategy described in Sec. 2. While in [8] these parameters are determined through an inverse optimization procedure, in this paper we will set them to $100 \mathrm{Nm} / \mathrm{rad}$ for the evaluation. Note however 


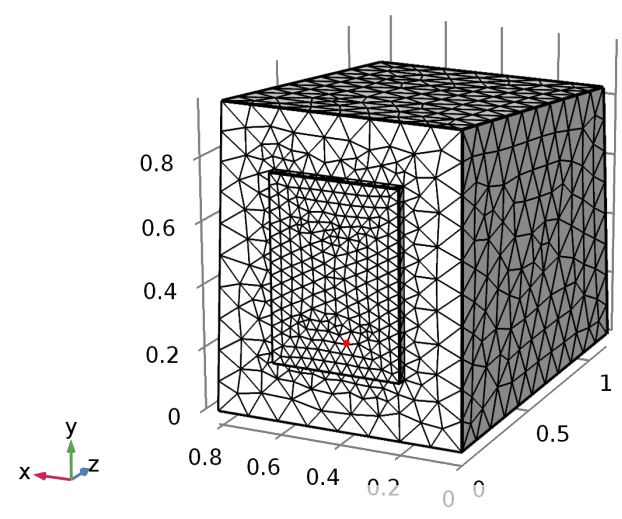

Figure 1: Mesh of the soundbox. The excitation point is indicated with a red dot.
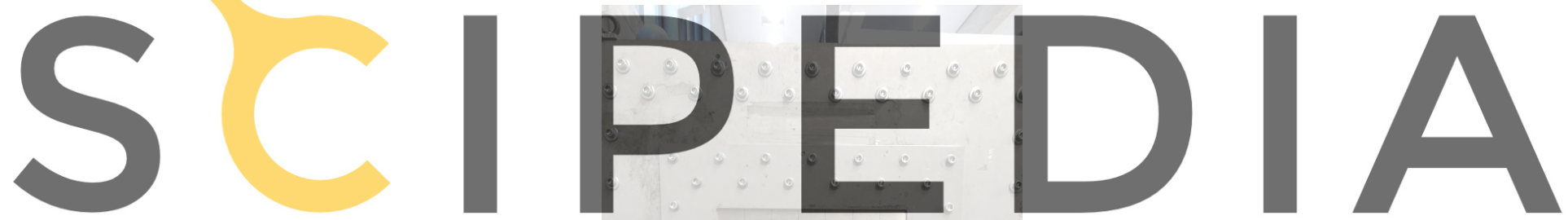

Register for free at https//www.scipedia.com to download the version without the watermark

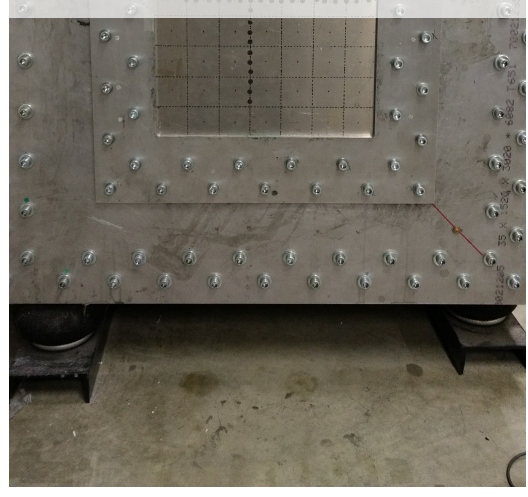

Figure 2: The soundbox. 

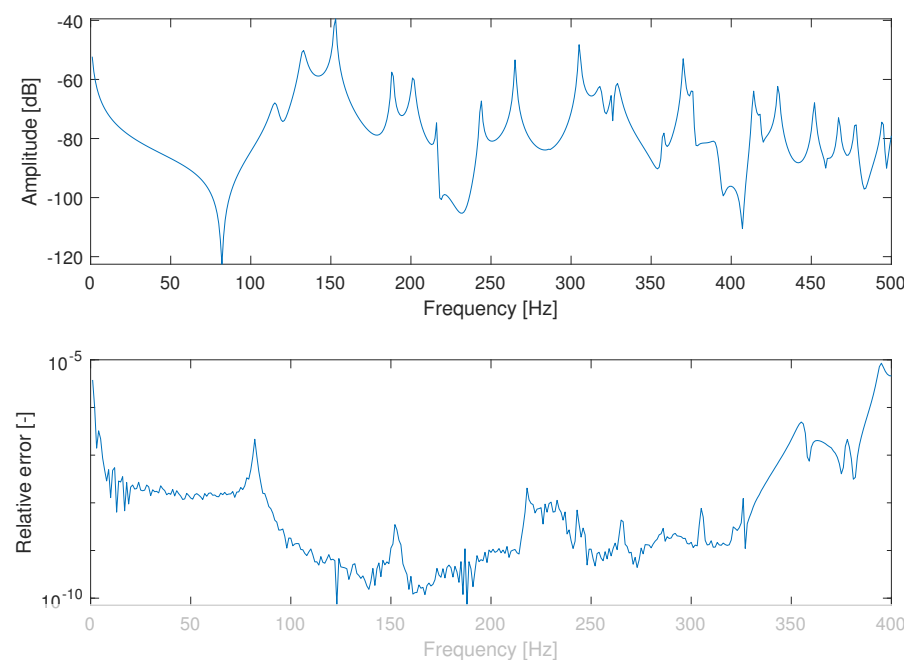

Figure 3: FRF and relative error of previous approach.

that for the construction of the ROM with the automatic reduction algorithm we choose random values of these parameters throughout the algorithm. The results are compared to the approach used in [1].

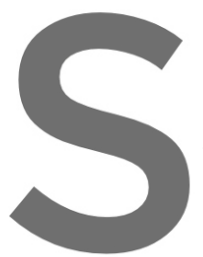

In Figure 3 the results of

the FRF from the ROM

interest (1-400 Hz) is set to

be seen that the resulting

error seems to be much

estimation procedure are shown
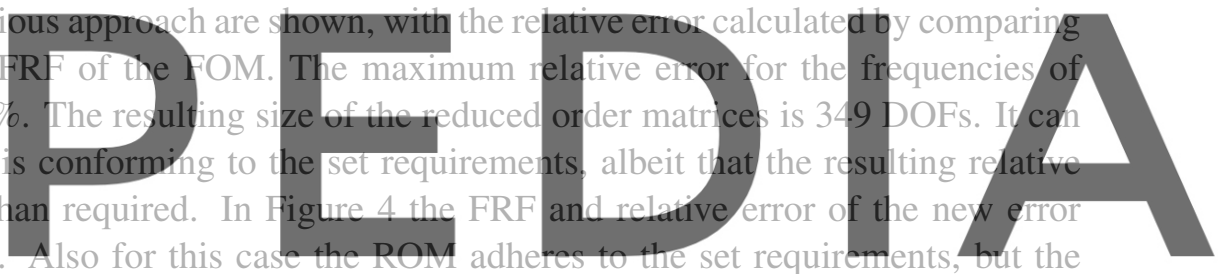

model size has significantly reduced to 254 DOFs. It can be seen that the relative error per frequency

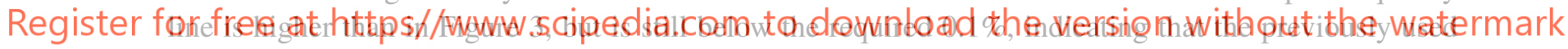
error definition was indeed too strict for these types of systems.

\section{CONCLUSIONS}

A disadvantage of most parametric model order reduction schemes is the need to sample the expected parameter space up front. Especially when the parameter range is unknown during model construction or when many parameters are considered this leads to a high off-line computional cost. However, when the parameters represent low-rank contributions to the system, such as is the case for parameter fluctuations related to boundary conditions, local mass loading due to heavy sensors, etc. it is possible to rewrite the system to a form in which non-parametric model order reduction techniques can be used instead, thus omitting the parameter sampling requirement. In this paper a new formulation is shown for second order systems that results from a finite element discretization of a vibro-acoustic system. The consequence of rewriting the system to an non-parametric form is that extra input vectors are obtained. Since the resulting reduced basis for calculating the reduced order model is dependent on the amount of independent inputs, this means a potentially large reduced order model. However, when these inputs are closely placed, the response might be similar between the inputs, leading to a possibly smaller reduced order model. 

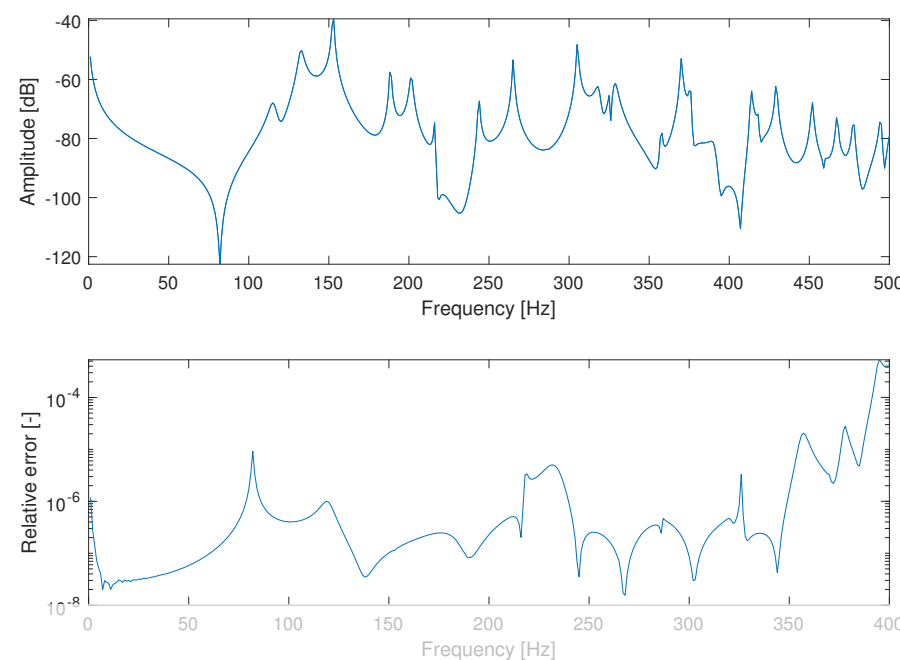

Figure 4: FRF and relative error of current approach.

To calculate the reduced basis an automatic Krylov based strategy is followed. Two approaches for calculating the reduced basis are compared, of which one of them is introduced in this paper. The first approach decides the chosen expansion points and amount of matched moments by estimating the enror of each input individual parameter fluctuations 1 ha looking at a random se approach leads to a signi user.
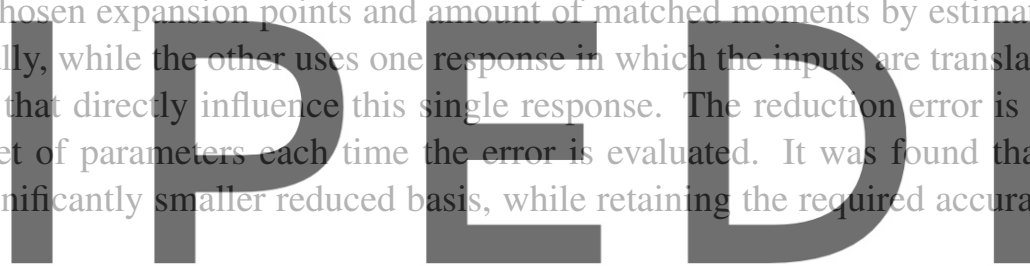

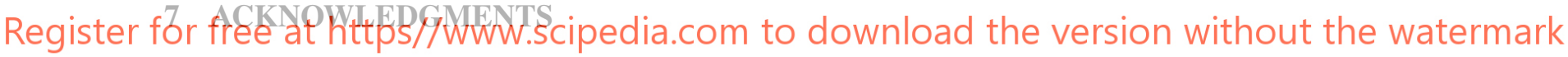

The research of S. van Ophem (fellowship no. 1277021N) is funded by a grant from the Research

Foundation - Flanders (FWO). The Research Fund KU Leuven is gratefully acknowledged for its support.

\section{REFERENCES}

[1] S. van Ophem, E. Deckers, W. Desmet, Parametric model order reduction without a priori sampling for low rank changes in vibro-acoustic systems, Mechanical Systems and Signal processing, (2019) 130: 597-609.

[2] S. van Ophem, A. van de Walle, E. Deckers, W. Desmet, Efficient MIMO Krylov subspace model order reduction for vibro-acoustic systems, in: ANTARES - Advanced Training and Research in Energy Efficient Smart Structures, KU Leuven, Leuven, Belgium, (2018).

[3] S. Gugercin, A. Antoulas, C. Beattie, $\mathcal{H}_{2}$ Model Reduction for Large-Scale Linear Dynamical Systems,SIAM Journal on Matrix Analysis and Applications, (2008), 30:609-638.

[4] P. Benner, S. Gugercin, K. Willcox, A Survey of Projection-Based Model Reduction Methods for Parametric Dynamical Systems, SIAM Review 57, (2015), 4:483-531. 
[5] S. van Ophem, O. Atak, E. Deckers, and W. Desmet. Stable model order reduction for time-domain exterior vibro-acoustic finite element simulations, Computer Methods in Applied Mechanics and Engineering, (2017) 325:240-264.

[6] U. Baur, C. Beattie., P. Benner, Mapping parameters across system boundaries: parameterized model reduction with low rank variability in dynamics, Proceedings in Applied Mathematics and Mechanics, (2014) 14 (1):19-22.

[7] Y. Yue, K. Meerbergen, Parametric model order reduction of damped mechanical systems via the block Arnoldi process, Applied Mathematics Letters,(2013), 26:643-648.

[8] S. van Ophem, A. van de Walle, E. Deckers, and W. Desmet. Efficient vibro-acoustic identification of boundary conditions by low-rank parametric model order reduction, Mechanical Systems and Signal Processing, (2018) 111:23-35.

[9] A. van de Walle, The power of model order reduction in vibroacoustics. $\mathrm{PhD}$ thesis, KU Leuven, Leuven, Belgium, 2018.

[10] C. Beattie, S. Gugercin, Z. Tomljanovic, Sampling-free parametric model reduction of systems with low-rank parameterization, Advances in Computational Mathematics, (2020) 46:83

[11] Z. Bai, Y. Su, Dimension Reduction of Large-Scale Second-Order Dynamical Systems via a SecondOrder Arnoldi Method, SIAM Journal on Scientific Computing 26, (2005), 5:1692-1709.
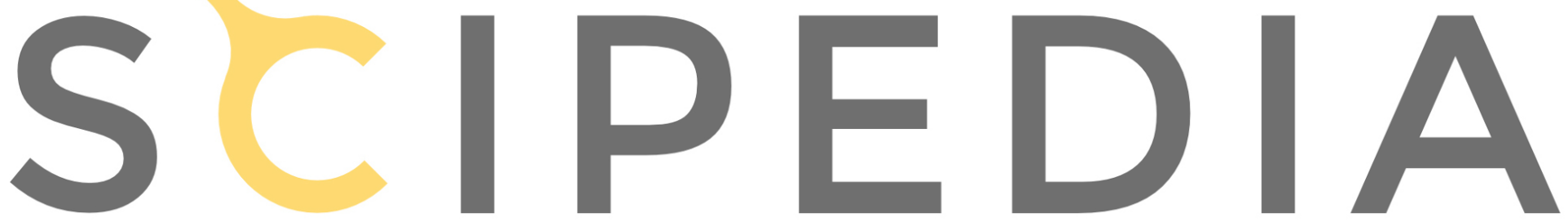

Register for free at https//www.scipedia.com to download the version without the watermark 